\title{
Investigation of Precipitation and Segregation of Secondary Phase Byproducts in Intermetallic Superconducting Materials
}

\author{
Yi-Feng Su${ }^{1}$, Shreyas Balachandran ${ }^{1}$, Yesusa Collantes ${ }^{1,2}$, Fumitake Kametani ${ }^{1,2}$, Chiara Tarantini ${ }^{1}$, \\ Peter J. Lee ${ }^{1}$, Eric E. Hellstrom ${ }^{1,2}$ and David C. Larbalestier ${ }^{1,2}$ \\ 1. National High Magnetic Field Laboratory, Tallahassee, FL, USA. \\ 2. FAMU/FSU College of Engineering, Tallahassee, FL, USA.
}

For broader acceptance, the next generation of superconductors for high magnetic field applications will need to be both higher performance and lower cost. Here we contrast two superconductors that are candidates for high field magnets; $\mathrm{Nb}_{3} \mathrm{Sn}$, which has a long history but has seen renewed interest in recent years because of remarkable advances in its properties, and Fe-based superconductors that offer potentially low-costs and very high fields. TEM techniques are essential to understand how to engineer the desired micro-/nanostructure that ultimately defines the properties for these superconductors.

For $\mathrm{Nb}_{3} \mathrm{Sn}$, the recent advances have been due to modifications that have greatly increased the density of nanostructural features that are needed to pin the flux vortices at high field. We have fabricated Ultra Fine Grain (UFG) $\mathrm{Nb}_{3} \mathrm{Sn}$ monofilament conductor using Hf additions that can now carry much higher currents at high field [1]. Using TEM we show the presence of nanoparticle precipitation within the $\mathrm{Nb}_{3} \mathrm{Sn}$ grains that are not found without the Hf addition. These precipitates are not observed in $\mathrm{Nb}-\mathrm{Ta}-\mathrm{Hf}$ alloy from which the $\mathrm{Nb}_{3} \mathrm{Sn}$ is formed by heat treatment. The significance of the precipitates is not clear at present as they are too few in number to directly the large performance gains. Fig. 1a shows a representative TEM bright field image of the $\mathrm{Nb}_{3} \mathrm{Sn}$ region. It appears that those dark contrast (white arrows) within the $\mathrm{Nb}_{3} \mathrm{Sn}$ grains corresponding to nanoparticle precipitation. Fig. 1b shows the EDS spectra taken from grain and precipitate, respectively. It indicates grain and precipitate have the same compositions with negligible amount of $\mathrm{O}$ and undetectable Hf. The EDS quantification (inset) shows the precipitate has relative higher amount of $\mathrm{Nb}$ and lower amount of $\mathrm{Sn}$ compare to the grain. Initial analysis indicates that the particles are Sn deficient but efforts are underway to prepare site-specific samples for a more precise evaluation.

Compared to $\mathrm{Nb}_{3} \mathrm{Sn}$, K-doped $\mathrm{BaFe}_{2} \mathrm{As}_{2}(\mathrm{~K}-\mathrm{Ba} 122)$ has 2 times higher $T_{\mathrm{c}}(39 \mathrm{~K})$ and 3 times larger $H_{\mathrm{c} 2}(\sim 90 \mathrm{~T}$ at $4.2 \mathrm{~K})$ [2,3]. Also, unlike the commercial high temperature superconducting (HTS) cuprate conductors, K-Ba122 uses inexpensive $\mathrm{Ba}, \mathrm{Fe}$, and $\mathrm{As}$ (and $\mathrm{K}$ as a dopant) constituents which can be processed into the wire form with an inexpensive metal sheath. Such advantages make Ba122 very attractive as a high field conductor with which significant cost reduction of the high field magnets would be possible [2]. We utilized STEM-EDS to analyze the chemical variation between grains and at the grain boundaries of samples. Fig. 2a represents TEM bright field images of K-Ba-122 with $50 \mathrm{~nm}$ estimated grain size on average. Fig. 2b shows STEM-ADF (annular dark field) image of a standard K-Ba-122 where significant compositional variations can be seen at the grain boundaries and triple junctions. Dark regions in ADF image signal large stoichiometry deviations. STEM-EDS 2-D maps in Fig. 2c shows that the segregation at triple junctions is mainly composed of Ba along with large amounts of oxygen, and grain boundaries are deficient in $\mathrm{K}, \mathrm{Fe}$ and $\mathrm{As}$ and Ba-rich. We believe the findings to be helpful in understanding the microstructure of $\mathrm{Nb}_{3} \mathrm{Sn}$ and Fe-based superconductors and their potential effects on performance [4]. 
References:

[1] S Balachandran et al., Supercond. Sci. Tech. (2019). DOI: 10.1088/1361-6668/aaff02

[2] J Weiss et al., Nat. Mater., 11 (2012), p. 682.

[3] Y Collantes, PhD Dissertation (2018).

[4] This work was supported by the U.S. Department of Energy, Office of Science, Office of High Energy Physics under Award Numbers DE-SC0012083 and DE-SC0018750, the National Science Foundation under Cooperative Agreements NSF-DMR-1157490 and DMR-1644779 and the State of Florida.
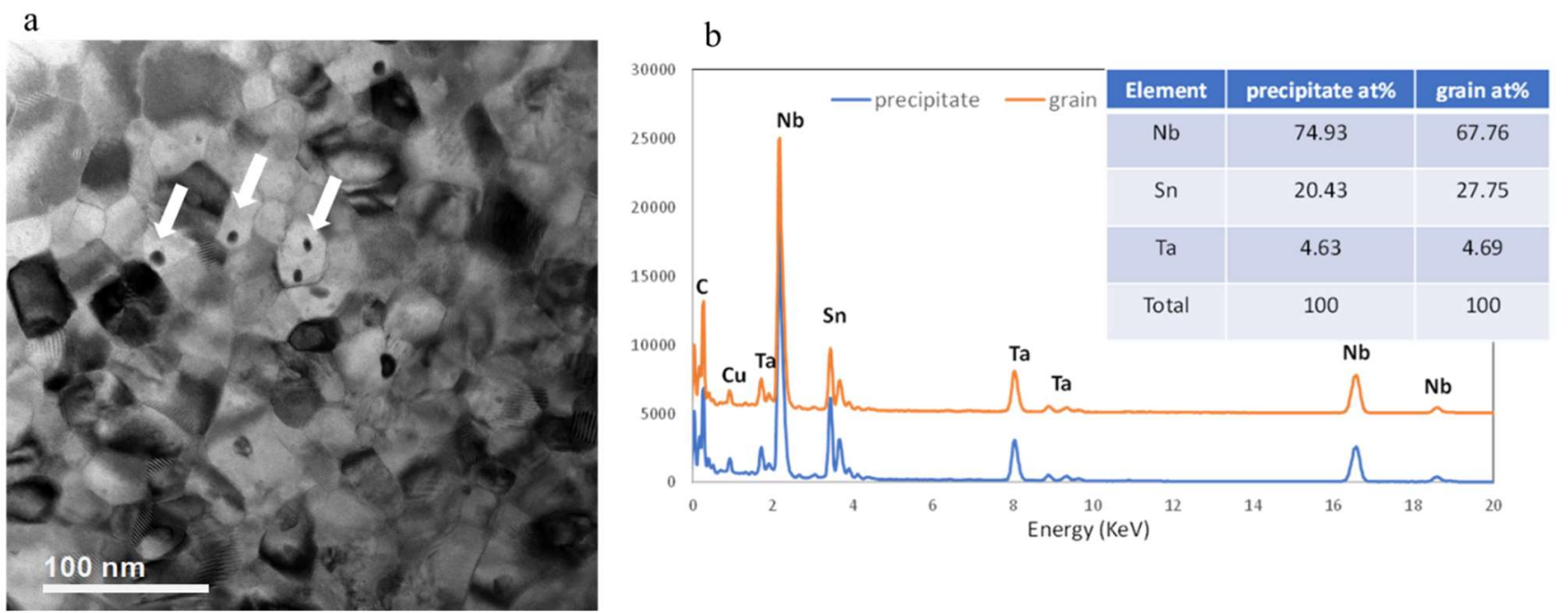

Figure 1. (a) TEM-BF image of $\mathrm{Nb}_{3} \mathrm{Sn}$ with intragranular nano-precipitates (white arrows). (b) EDS spectra and quantification compared for the grain and precipitate, respectively.

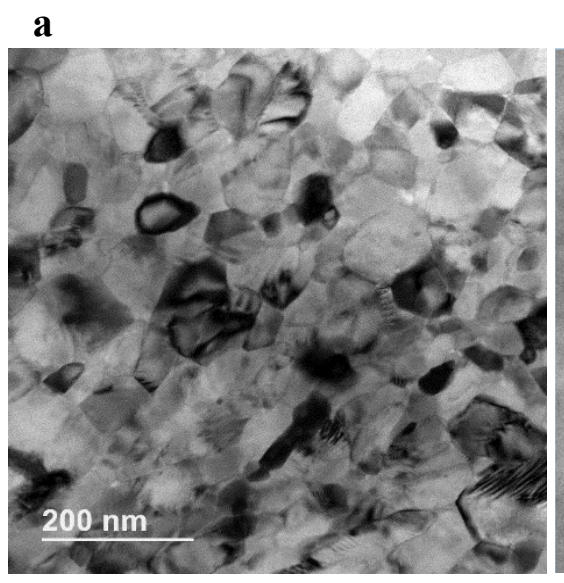

b

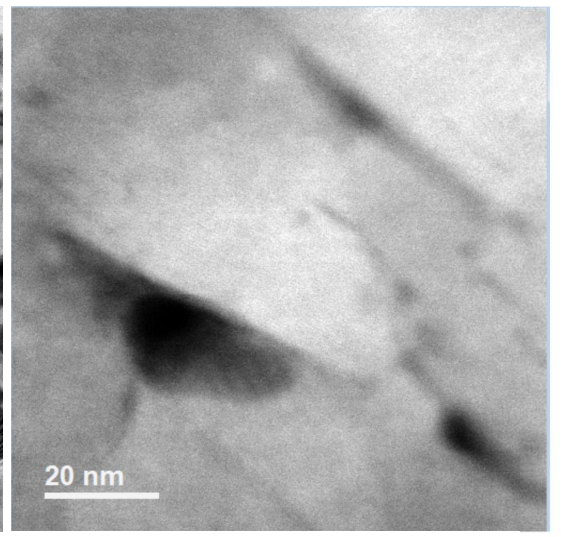

Figure 2. (a) TEM BF and (b) STEM BF images of K-doped $\mathrm{BaFe}_{2} \mathrm{As}_{2}$ (grain size of $\sim 50 \mathrm{~nm}$ ) shows element segregation at triple junction and grain boundaries. (c) STEM-EDS 2-D map displaying the elemental distribution across the entire region in (b). Barium along with oxygen is observed at the triple junction and grain boundary
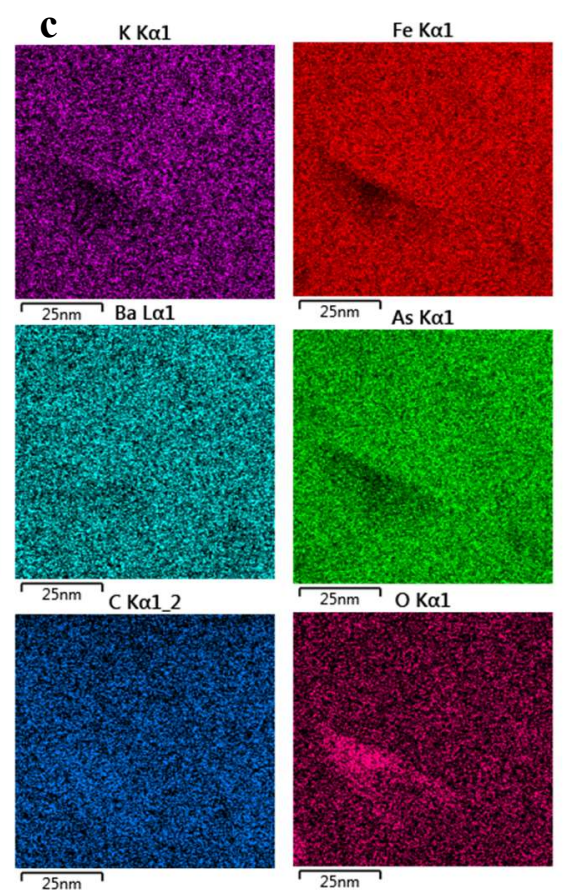